\title{
Experimental models of neurological disease: neuropathology determines what is virtual reality, science or fiction
}

\author{
Werner Paulus ${ }^{1}$
}

Published online: 10 January 2017

(C) Springer-Verlag Berlin Heidelberg 2017

This issue of Acta Neuropathologica includes a cluster of six review articles to highlight how experimental models relate to neurological diseases in humans. The topics are Alzheimer's disease (AD), amyotrophic lateral sclerosis (ALS)/frontotemporal lobar degeneration (FTLD), prion disease, multiple sclerosis, ischemic stroke and gliomas. These disorders are amongst the most common causes of neurological disability and death all over the world, posing huge medical, psychological, social and economical burden and of course they present major scientific challenges.

In order to better understand the underlying mechanisms and to find more efficient treatments for these diseases, experimental models are essential. Many models have been established over several decades, such as transgenic models of neurodegenerative disorders, sophisticated stroke models, various types of experimental autoimmune encephalomyelitis and in vitro and in vivo models of brain tumors. At the same time, the (non-medical) neuroscience community has increasingly developed an interest in neurological disease. This has led to new insight into disease mechanisms, and exciting new methodologies have been applied also to neurological research.

However, despite or because of this excitement it is often overlooked that experimental animals do not simply have the disease, and this is reflected in the contributions to this cluster. For example, A $\beta$ plaques of transgenic AD models resemble senile plaques of human $\mathrm{AD}$ brains, whereas the fine structure and molecular composition (A $\beta$ species, TDP-43, etc.) are different. Furthermore, other features of

Werner Paulus

werner.paulus@uni-muenster.de

1 Institute of Neuropathology, University Hospital Münster, Pottkamp 2, 48149 Münster, Germany human $\mathrm{AD}$ are usually missing in transgenic models, such as tau pathology, synaptic impairment, neuronal loss, glial pathology, white matter and vascular aberrations as well as macroscopical brain atrophy. Tau transgenic mice successfully recapitulate tau hyperphosphorylation and its neuronal accumulation and microglia reaction, but they also typically show motor neuron and memory deficits, features that are not prominent in the corresponding human disease, FTLD-tau. The photothrombosis model of stroke produces reproducible infarcts of predetermined size and location in rodents, whilst the lack of an ischemic penumbra and the type of brain edema are in contrast with stroke in human brains. Human gliomas show clinically relevant typical features concerning genetics, intratumoral heterogeneity and cellular interactions within the brain, but no glioma model sufficiently reflects all of these features. These discrepancies are among the reasons why clinical translation of therapies that proved successful in animal models has been usually frustrating.

There is no ideal model and a model is just a model. It is clear that models cannot perfectly reflect all aspects of human disease due to major differences between animals and men. These differences include neuroanatomy, cellular and molecular features of the nervous system, the cell types involved in pathology (for example, due to the promoter driving transgene expression), the immune system, life span that may be too short for developing typical pathology in animal models, as well as differences in age and comorbidities, among others. A significant effort is made to refine the methodologies and to establish models that better reflect human pathophysiology, such as knock-in models of AD where humanized mutated APP is expressed in correct cell types at "physiological" levels. Nevertheless, despite the known limitations, good models are of course 
essential as they allow for a modeling of selective features under highly controlled conditions.

A number of measures compensate for limitations of disease models. For example, experimental studies may make use of more than one model, each of them exploring different aspects of human disease, or a transgenic model may be tailored to address specific hypotheses, as outlined in this issue by Brandner and Jaunmuktane on prion diseases. Translational conclusions about human disease are limited by the specific model used. For example, experiments with mutant SOD1 rodent models (which are most often used in ALS research) allow conclusions only for human ALS with SOD1 mutation which, however, represents less than $2 \%$ of all sporadic cases of ALS.

To increase the translational value of animal studies, experimental data need to be validated on human tissues in order to reduce the likelihood of studying experimental artifacts that have no clinical relevance. In many developed nations experimental research on neurological disease is relatively well funded. On the other hand, there is a remarkable neglect by funding bodies of the value and importance of brain banks and adequately characterized human brain tissues. It is somewhat disconcerting that neuropathological studies sometimes are considered "descriptive", a term that has become increasingly depreciative, to delineate them from "mechanistic" studies. Needless to say that this is nonsensical, because any experiment requires careful description. "Collecting" human tissues is anything but trivial and requires expert knowledge in anatomy, neuropathological diagnostic criteria, ethical and legal issues. Ignoring the value of human tissues in basic research can significantly compromise validity and quality of "experimental" research. Even worse, since the direction of research tends to follow money (and impact points and prestige), and the pressure exerted on researchers for producing glory and popularity is increasing, even some neuropathologists paradoxically lose interest on research using human tissues.

Challenges exerted by neurological disorders are too massive, life is too short and money is too sparse to undertake and believe in irrelevant experiments. Neuropathology, being furnished with the greatest expertise at the crossroads of experimental neuroscience and human neurological disease, is in a unique position to adjust experimental results to reality, i.e., adequately comparing experimental findings with human pathology. What is needed are more neuropathologists who can support and advise neuroscientists with limited expertise in neurological disease, and more experimental neuroscientists who are aware of the importance and strength of neuropathology for validating their research. Hopefully the present cluster of articles, written by authors who are experts in both human neuropathology and experimental neuroscience, will help to move the field forward in this direction. 